\title{
Application of two arrays of 32 IR detectors: The thermal diffusivity measurement on anisotropic materials
}

\author{
By Didier Demange, Pierre Beauchêne, Michel Bejet
}

ONERA - DMSC - Fort de Palaiseau, Chemin de la Hunière 91761 Palaiseau, France CEDEX Tel: 33 (0) 1699361 23, Fax: 33 (0) 1699361 25, E-mail:demange@onera.fr

\begin{abstract}
Two arrays of $32 \mathrm{IR}$ detectors are used for the thermal diffusivity measurement on composite materials in thin plate. The method consists of illuminating with a short pulse one of the faces of a square sample heterogeneously, to be able to analyze heat transfers in parallel and perpendicular directions of the faces of the specimen.
\end{abstract}

\section{Introduction}

An example of use of two arrays of 32 IR detectors is showed in this article. The application is the thermal diffusivity measurement on anisotropic materials in thin plate. The use of composite fibber materials for application in aerospace system required developing a method for characterizing thin plate composites in the three main directions of the material. The apparatus, which requires only one test specimen, allows measurements to be made without cutting and reconstituting specimens.

The method consists of illuminating one of the faces of a square sample with a heterogeneous energy pulse (two parallel bands separated with a non-irradiated zone). So, we are able to analyze heat transfers in parallel direction ( $x$ and $y$ directions), and perpendicular direction (direction $z$ ) of the faces of the specimen. It therefore allows characterization of thin plate composite materials in which the fiber distribution differs in the three directions.

To be able to make measurements on a composite, the composite must be able to be assimilated to an homogeneous medium or be homogenisable. Generally, measurements on a non-homogenisable composite lead to poor agreement between theoretical and experimental thermograms. In this case, it is necessary to increase the thickness crossed by the thermal wave. The tridirectional flash method is therefore advantageous since the measurement thickness taken into account is approximately equal to half the specimen width, i.e. approximately 10-mm.

Experimental developments have been integrated an ONERA diffusimeter using a phosphate glass laser source. The operating temperature range of the device varies from $20^{\circ} \mathrm{C}$ or $100^{\circ} \mathrm{C}$ to $2000^{\circ} \mathrm{C}$.

\section{1. principle}

The sample has a square base measuring $L$ on a side and a thickness $e$ (figures 1 and 2). The pulse width is assumed infinitely short. The sample is illuminated in two rectangular regions with length $L$ and equal width $a$, separated by an unilluminated region of width $L-2 a$. 
The system to be solved is: $\lambda_{x} \frac{\partial^{2} \theta}{\partial x^{2}}+\lambda_{y} \frac{\partial^{2} \theta}{\partial y^{2}}+\lambda_{z} \frac{\partial^{2} \theta}{\partial z^{2}}=\rho c \frac{\partial \theta}{\partial t}$. Simulations have shown that an analytic model ignoring the losses or taking only the axial losses into account is insufficient for identifying the diffusivity in $x$ direction with sufficient accuracy. We therefore used an analytic model, which takes into account both the facial and the lateral losses. Heat transfer with the outside is characterized by a heat exchange coefficient $h_{m}$ common to all faces and constant over time. The maximum temperature rise is less than 5 degrees $C$, so during the transient state, proprieties are supposed independent of the temperature. The solution of the transient state $\left(T=\theta-\theta_{\infty}\right)$ is obtained as an equation with separate variables in $x, y$ and $z$, with the form: $T(x, y, z, t)=F(x, t) . G(y, t) . H(z, t)[2]$.

\section{Identification of the Diffusivity in the $z$ Direction}

The diffusivity in the $z$ direction is obtained by integrating the temperature signals on the rear face $\left(z=e, y=\frac{L}{2}\right)$ by the equation $\phi(t)=L \int_{0}^{L} T_{\exp }(x, t) d x$.

Yielding, for the experimental thermograms:

$$
\phi(t)=L \sum_{i=1}^{i=32} T\left(x_{i}, t\right) \Delta x
$$

$T=$ temperature rise,$i=$ pixel number, $x_{i}=$ position of pixel $i$

It can be seen and demonstrated that the variation of this signal is independent of the energy distribution of the laser beam on the front face, provided there are no lateral losses. Under these conditions, the axial diffusivity can be obtained by a conventional identification technique used for isotropic materials after integration of the thermograms.

\section{Identification of the Diffusivity in the $x$ Direction}

Identification of the diffusivity in the $x$ direction consists in taking the ratio of two thermograms for two positions, $x_{i}$ and $x_{j}$, on the specimen, i.e. $\mathfrak{R}\left(x_{i}, x_{j}, t_{x}^{*}\right)=\frac{F\left(x_{i}, t_{x}^{*}\right)}{F\left(x_{j}, t_{x}^{*}\right)}$ with reduced temperature $t_{x}^{*}=\frac{\alpha t}{L^{2}}$.

This yields for two positions, $x_{i}$ and $x_{j}$, an expression of the diffusivity independent of the axial diffusivity and the characteristics of the temperature detector.

The diffusivity is then identified by the equation:

$$
\alpha_{x}\left(t_{n}\right)=\frac{\mathfrak{R}^{-1}\left\{R_{\text {exp }}\left(t_{n}, x_{i}, x_{j}\right)\right\} L^{2}}{t_{n}}
$$

for $i, j$ pairs corresponding to regions illuminated on the specimen such that the experimental temperature ratios $R_{\exp }\left(t, x_{i}, x_{j}\right)$ are significantly different from $1 . x_{i}$ is near the center of the sample and $x_{j}$ on the edge.

For each thermogram positioned at $x_{i}$, we have the mean diffusivity given by: 


$$
\alpha\left(x_{i}\right)=\frac{\sum_{N_{\min }}^{N_{\max }} \alpha\left(t_{n}\right)}{\left(N_{\text {max }}-N_{\text {min }}\right)+1}
$$

The final diffusivity is given by:

$$
\bar{\alpha}=\frac{\sum_{i=I_{\min }}^{i=I_{\max }} \alpha\left(x_{i}\right)}{\left(I_{\max }-I_{\min }\right)+1}
$$

To take thermal losses into account, an average loss term $h_{m}$ is determined from the integrated thermogram during the cooling phase. The loss term $h_{m}$ is adjusted until a satisfactory agreement is obtained between the theoretical and experimental thermograms

This identification method was validated by simulation of thermograms obtained by finite differences.

About ten thermograms in the center of the sample are used to determinate the diffusivity in the $x$ direction. To check results, we have to control the constancy of the equation (2) with the time and the constancy of equation (3) with the position of the detector. If the position of the array is not know with accuracy, the equation (3) is not constant. However, if the ten thermograms are in the center of the sample, the mean value is not too wrong, because one value is compensated by the value got in symmetrical position.

\section{Experimental Developments}

Experimental developments were integrated an ONERA diffusimeter using a phosphate glass laser source.

The items specific to measurements on a 3D composite are a device providing an accurate image of the specimen on an array of 32 IR detectors centered and a graphite specimen-holder equipped with an occultation piece (figure 4).

Two pyrometers sighting several different regions on the rear face of the specimen were designed and built (figures 3 and 6). Their operating temperature range is between $20^{\circ} \mathrm{C}$ and $2000^{\circ} \mathrm{C}$. It was designed to guarantee positioning of the sighted regions on the specimen with respect to the laser impact. The detection system consists on an array of 32 InSb IR detectors centered on $5 \mu \mathrm{m}$ for low temperature measurements or an array of 32 germanium IR detectors centered on $1.3 \mu \mathrm{m}$ for high temperature measurements. An optical system for each array whose magnification allows complete observation of a half specimen. The array of $32 \mathrm{InSb}$ IR detectors is $15,65 \mathrm{~mm}$ long; each detector is $0,35 \mathrm{~mm}$ large and 1 $\mathrm{mm}$ high. The array of $32 \mathrm{Ge}$ IR detectors is $16 \mathrm{~mm}$ long, each detector is $0,45 \mathrm{~mm}$ large and $1 \mathrm{~mm}$ high. An original card was built to amplify the $2 \times 32$ detectors signals and to compensate the offset signal before the pulse laser, the principle is given figure 5 . Software written in Visual basic controls the device and gets data from the amplifiers with an acquisition card and save them in a hard disk. The acquisition card has a $330 \mathrm{KHz}$ frequency of measurement, so each detector is acquired with a $10 \mathrm{kHz}$ frequency. The electronic device allows setting the gain of each detector at the same value, so each line has the same relation between the signal and the temperature and the ratio of two thermograms are calculated with a good accuracy. Each array is positioned on a motorized translation system, whose move allows a precision positioning of detectors in relation with the sample image. 
An original graphite specimen-holder equipped with an occultation piece was built. This piece has two parallel holes of five millimeter large and 20 millimeter high separated with a 10-millimeter large closed zone (figure 4).

The measurements made by this apparatus have accuracy similar $(\approx 3 \%)$ to those made conventionally by the « flash » method. For a homogeneous material, where we must have $\alpha_{x}=\alpha_{z}$, the method supplies two diffusivity values, improving the measurement reliability and accuracy.

\section{Examples of results}

Measurements on Anisotropic Graphite were conducted between $100^{\circ} \mathrm{C}$ and $1600^{\circ} \mathrm{C}$ in an argon atmosphere. A square base specimen $(20 \mathrm{~mm}$ on a side) and two cylindrical control specimens (dia. $20 \mathrm{~mm}$ ) at 90 degrees from one another were taken from a graphite rod.

Figure 7 shows the results obtained on all the specimens. The measurements made using the 3D method (square specimen) are illustrated on the graph by symbols and those obtained using the conventional flash method are illustrated by solid lines (cylindrical specimens). We have a good agreement between the two methods. Figure 8 shows the thermograms got for an experiment at $600^{\circ} \mathrm{C}$ and the integrated thermogram calculated with the equation (1). Figure 9 shows for the same experiment, the diffusivity measurement got for each detector and each point, we can note the constancy of the diffusivity with time and position on the sample observed by the detectors.

A result on composite material is showed on figure 10. Measurements were conducted between $20^{\circ} \mathrm{C}$ and $1200^{\circ} \mathrm{C}$ in an argon atmosphere with a $2.5 \mathrm{~mm}$-thin sample.

\section{Conclusion}

An example of application of two arrays of 32 IR detectors has been exposed in this article. The two arrays have been applied for the measuring of the thermal diffusivity in the three principal directions on an anisotropic material. The advantages of the device are:

The three measurements on $x, y$ and $z$ direction are only made with one sample.

The completed characterization of one material is possible in one passage in temperature, which saves a lot of time.

The limited size of the sample allows to made measurements in a little furnace.

An example of results obtained on anisotropic graphite demonstrates the validity of the method. This method applied to an homogeneous material gives two thermal diffusivity values $\left(\alpha_{x}\right.$ and $\left.\alpha_{y}\right)$, thereby improving the measurement accuracy and reliability.

\section{REFERENCES}

[1] H.S. Carslay et J.C. Jeager, - Conduction of heat in solids. Clarendon Press, Oxford (1959).

[2] Didier Demange, Pierre Beauchène, Michel Bejet. Mesure des diffusivités axiale et radiale d'un composite $2 \mathrm{D}$ entre $500^{\circ} \mathrm{C}$ et $1500^{\circ} \mathrm{C}$. SFT Journée d'étude du 29 novembre 1995 
http://dx.doi.org/10.21611/qirt.2000.013

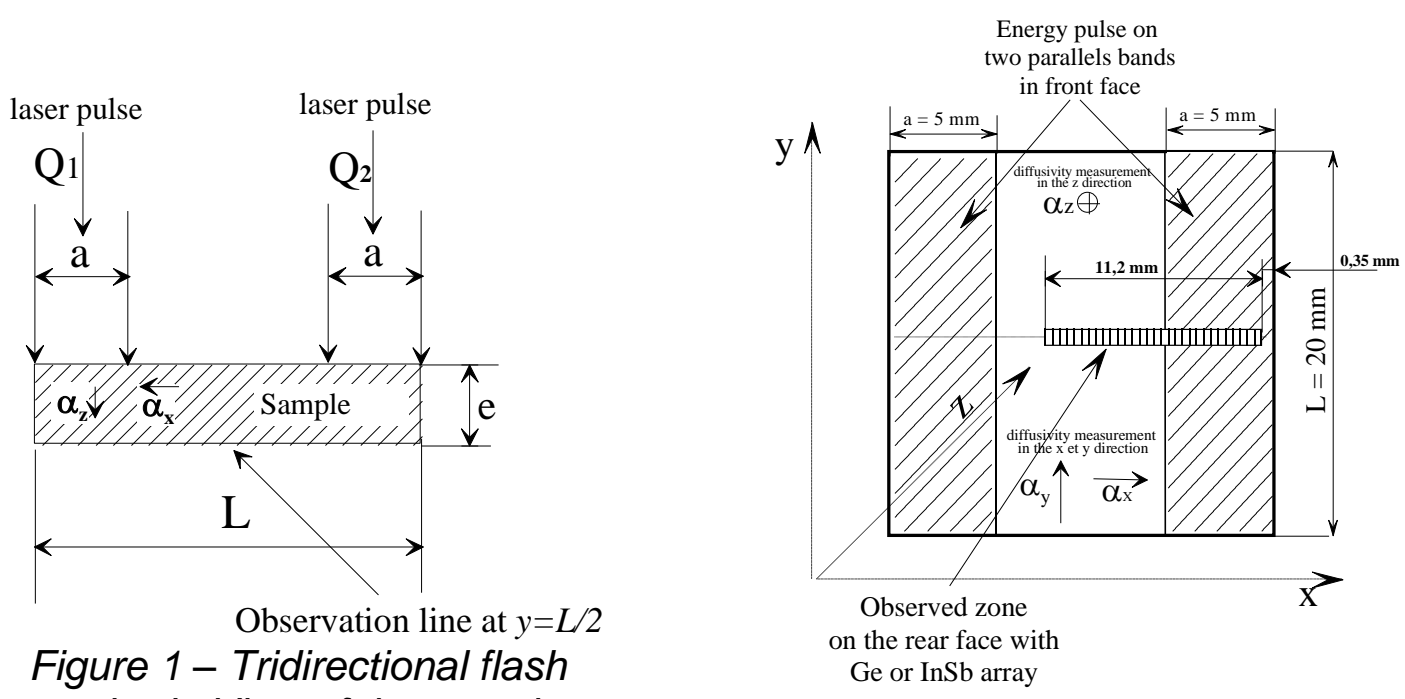

Figure 2 - Front face view of the sample

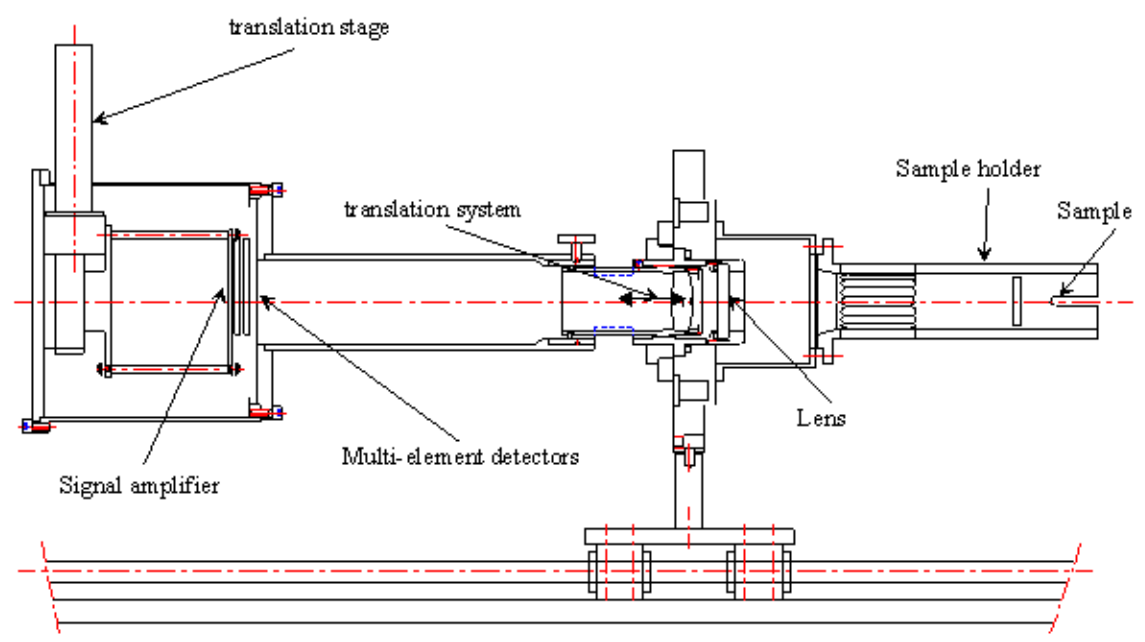

Figure 3-multidetector pyrometer

Sample

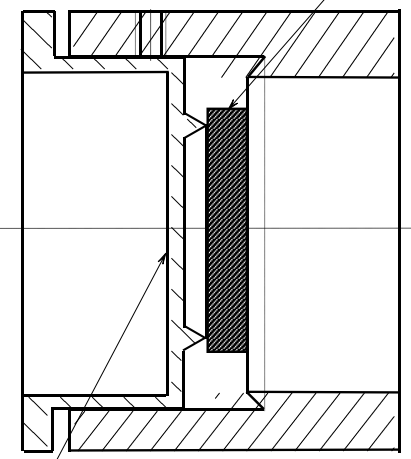

Ocultation piece

Figure 4 - Sample holder

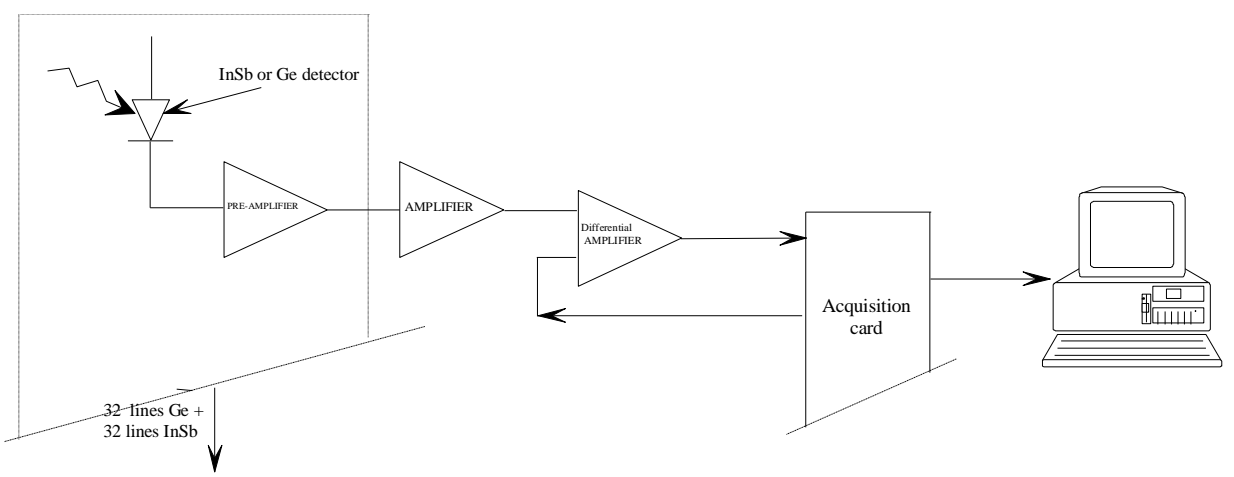

Figure 5-Electronic instrumentation 
http://dx.doi.org/10.21611/qirt.2000.013

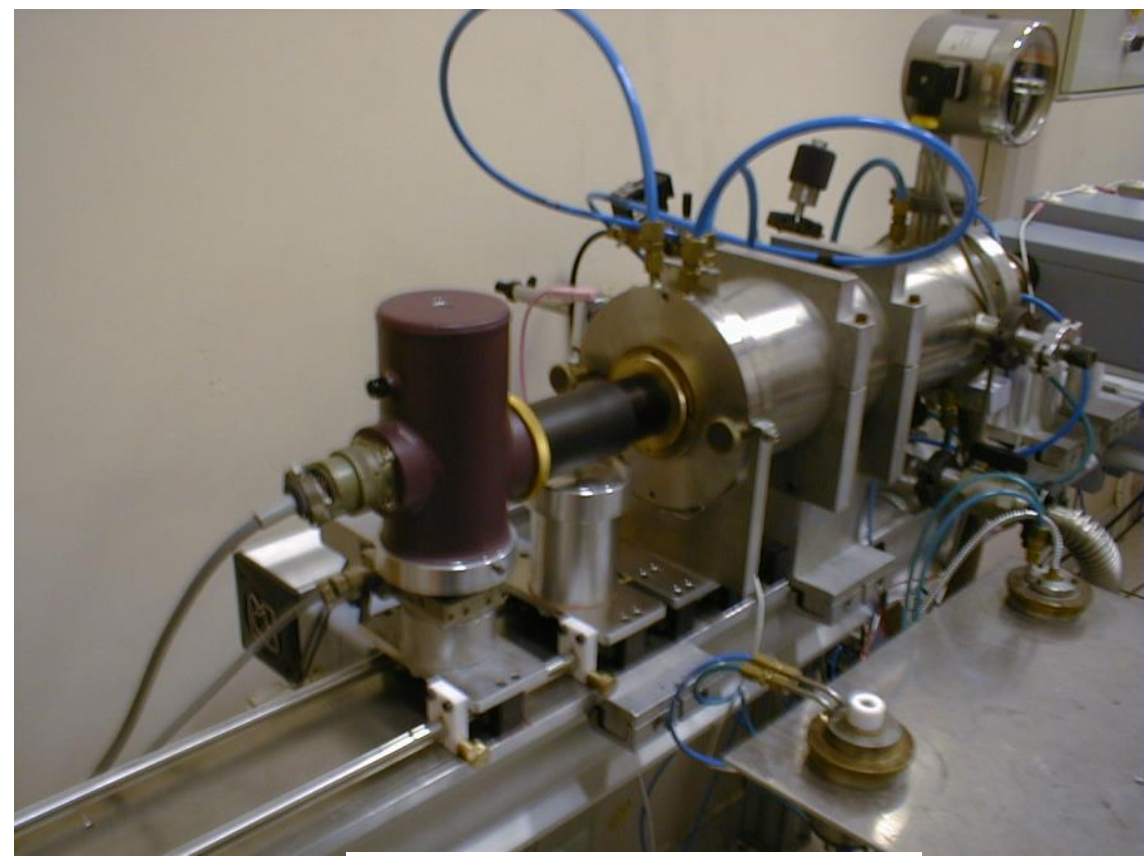

Figure 6-Device picture

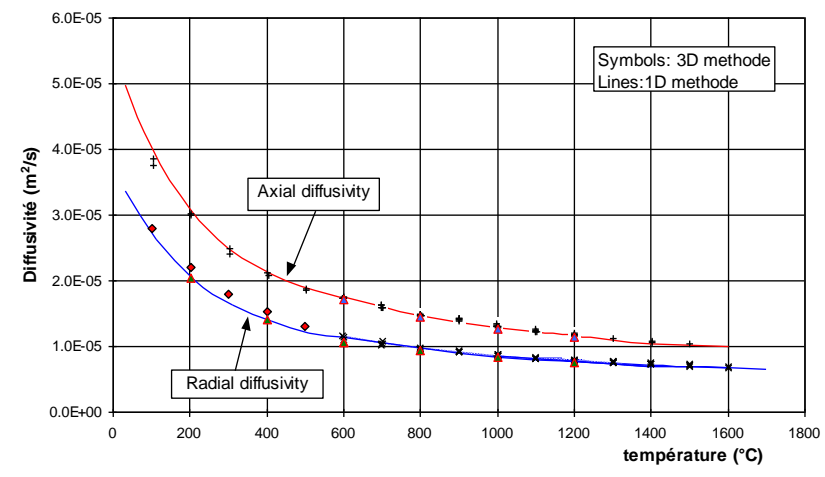

Figure 7- Results on an anisotropic graphite

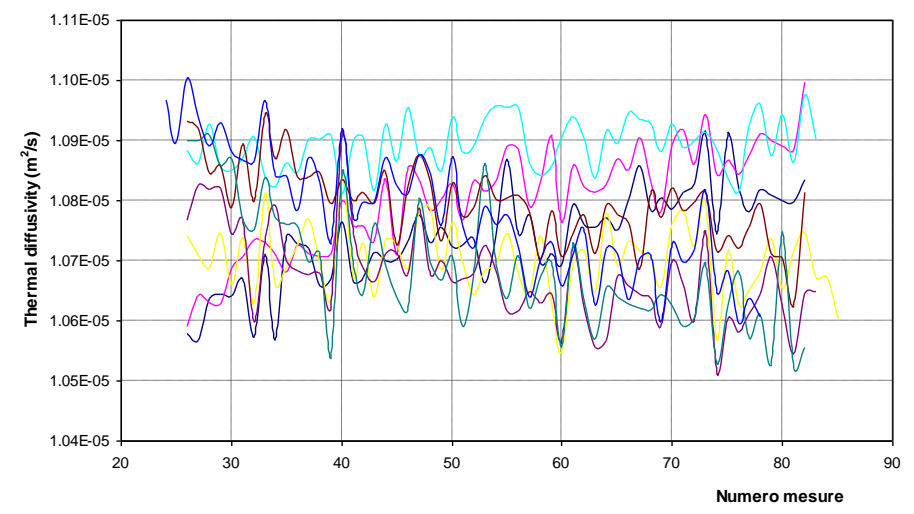

Figure 9 - Thermal diffusivity for each detector and each point of measurement (dilated scale)

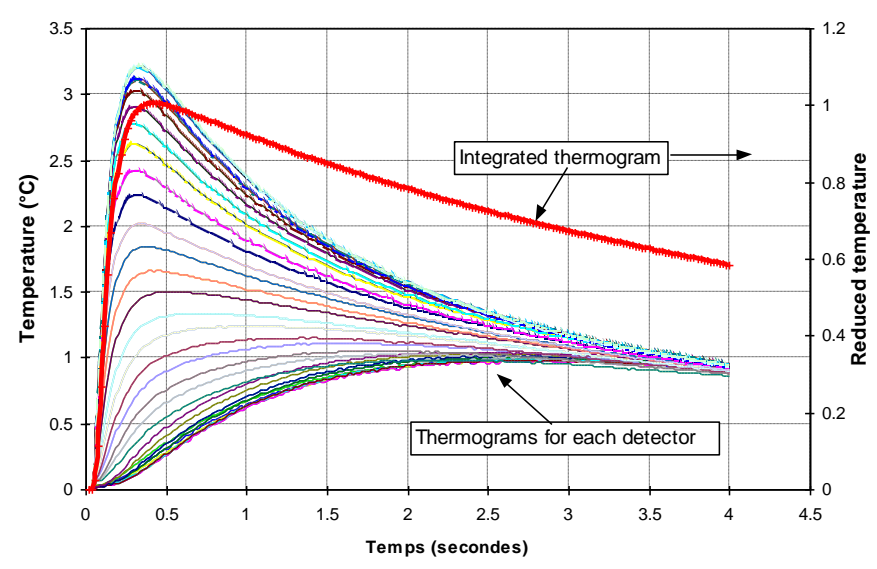

Figure 8 - Exemple of thermograms

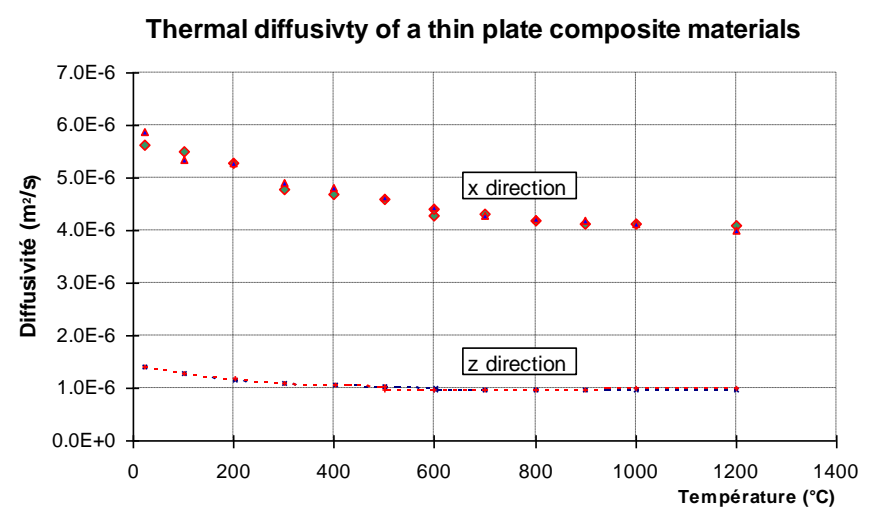

Figure 9 - Results on a composite material 